REVIEW

This paper is dedicated to the $70^{\text {th }}$ anniversary of the founding of Physiologia Bohemoslovaca (currently Physiological Research)

\title{
Is Apelin a New Biomarker in Patients With Polycystic Ovary Syndrome?
}

\author{
Ingrid DRAVECKÁ ${ }^{\mathbf{1}}$, Jana FIGUROVÁ ${ }^{\mathbf{1}}$, Ivica LAZÚROVÁ ${ }^{1}$ \\ ${ }^{1} 1$ st Department of Internal Medicine, Medical Faculty of P. J. Šafarik University, Košice, \\ Slovakia
}

Received April 21, 2021

Accepted September 7, 2021

\begin{abstract}
Summary
Polycystic ovary syndrome (PCOS) is associated with multiple risk factors for cardiovascular diseases, including insulin resistance, diabetes mellitus type 2, obesity, hypertension, and dyslipidaemia. Many studies have assessed the role of adipokines in the etiopathogenesis of PCOS, however, no single biomarker has been recognized to be in causal relation to the syndrome. Apelin has been identified as a new adipokine linked to obesity and insulin resistance. Some studies demonstrated that the apelin / apelin receptor could play a pivotal role in the pathogenesis of polycystic ovary syndrome, however the other yielded controversial results. Underlying mechanisms of possible involvement of apelin/apelin receptor complex are discussed.
\end{abstract}

\section{Key words}

Polycystic ovary syndrome • Adipokines • Apelin • Reproductive system • Cardiometabolic risk

\section{Corresponding author}

Ingrid Dravecká, 1st Department of Internal Medicine, Medical Faculty of P.J. Šafarik University, Košice, Trieda SNP 1, 04011 Košice, Slovakia. E mail: ingrid.dravecka@upjs.sk

\section{Introduction}

Polycystic ovary syndrome (PCOS) is the most frequent reproductive endocrinopathy in fertility age women. Although varying among different diagnostic criteria, its frequency ranges about $5-15 \%$ in the reproductive period (Goren et al. 2012, Choi et al. 2012,
Olszanecka-Glinianowicz et al. 2013). PCOS is characterized by hyperandrogenic state (clinical and/or biochemical), ovulatory dysfunction, infertility, and polycystic ovarian morphology on ultrasound. Hyperinsulinism and insulin resistance (IR) are common findings in PCOS women, and these traits have causeconsequence relationships with low-grade chronic inflammation and increased early atherosclerosis and cardiovascular disease (CVD) risk. Patients with PCOS have also long-term risks of dyslipidaemia, type 2 diabetes mellitus (T2DM), and endometrial cancer. In addition, more than $50 \%$ of patients have android type of obesity (Goren et al. 2012, Altinkaya et al. 2014). Moreover, women with PCOS exhibit an increased risk of pregnancy complications such as pregnancy-induced hypertension, pre-eclampsia, gestational diabetes, and premature birth (Hasan and Abd El Hammed 2018). Although IR and its metabolic consequences are not included in the diagnostic criteria of PCOS, they are generally accepted to play an important role in the pathogenesis of the disease (Goren et al. 2012).

The pathogenesis of PCOS is still unknown and seems to be multifactorial. Many studies have assessed the role of adipokines in PCOS development (Olszanecka-Glinianowicz et al. 2013). The link between disturbed adipokine secretion and IR in obesity has been established, and IR may represent one of the links between adipokines and polycystic ovaries (OlszaneckaGlinianowicz et al. 2013, Hasan and Abd El Hammed 2018). Adipokines, biologically active peptides secreted 
from adipose tissue, are involved in energy homeostasis and metabolism (Goren et al. 2012, Polak et al. 2017). It has been shown that tumour necrosis factor $\alpha$ (TNF- $\alpha$ ), interleukin 6 (IL-6), C reactive protein (CRP), insulinlike growth factor 1 (IGF-1), sex steroids, resistin, visfatin, adiponectin, and apelin are associated with IR (Goren et al. 2012). Disturbed secretion of adipokines may also impact the pathophysiology of PCOS through their effect on metabolism and sex steroid secretion (Saleh Ibrahim et al. 2018).

\section{Apelin}

\section{Structure and expression of apelin}

Apelin is a bioactive peptide originally identified from bovine stomach extracts as the endogenous ligand for an orphan receptor (APJ) and is found in several organs like heart, brain, kidneys, lungs, and adipose tissue (Goren et al. 2012, Olszanecka-Glinianowicz et al. 2013, Altinkaya et al. 2014, Sun et al. 2015). It appears to exert its effect by binding to the apelin receptor (APLNR) (Liu et al. 2020). APJ is a type of G proteincoupled receptor that was discovered based on its sequence similarly to angiotensin receptor (AT1). Apelin appears to carry out its effects by binding to the angiotensin receptor-like receptor (Liu et al. 2020, Kurowska et al. 2018). Apelin gene encodes a prepropeptide consisting of 77 amino acids, that is released into the circulation in several isoforms including apelin -12, -13, -16, -17, -19 and -36 (Altinkaya et al. 2014, Liu et al. 2020, Kurowska et al. 2018). Shorter forms of apelin (apelin-13) show much higher biological potency than the longer forms (apelin-36) (Kurowska et al. 2018).

\section{Physiology and pathology of apelin}

Hypothalamus and pituitary gland contain the primary sites of apelin action. In vitro study demonstrated that apelin-13 administration stimulated corticotropinreleasing hormone (CRH) and vasopressin release, suggesting that it may play an important role in the hypothalamic regulation of water intake and endocrine axis (Kurowska et al. 2018). Apelin increases adrenocorticotropic hormone (ACTH) secretion and decreases thyroid stimulating hormone (TSH), prolactin (PRL), follicle stimulating hormone (FSH), and luteinizing hormone ( $\mathrm{LH})$ secretion by the pituitary gland (Goren et al. 2012, Olszanecka-Glinianowicz et al. 2013, Altinkaya et al. 2014, Sun et al. 2015).
Moreover, apelin and APLNR are also implicated in several different key physiological processes, such as angiogenesis, cardiovascular system (CVS) in terms of hypotension, fluid homeostasis, cell proliferation, food intake and regulation of energy metabolism (Goren et al. 2012, Altinkaya et al. 2014, Sun et al. 2015, Liu et al. 2020, Kurowska et al. 2018). Apelin was found to be related to obesity and IR and its role in the regulation of glucose homeostasis has been suggested. Insulin stimulates the secretion of apelin, while apelin inhibits insulin secretion and stimulates glucose utilisation (Sun et al. 2015, Kurowska et al. 2018, Nayaker). Low circulating apelin concentrations have been associated with a higher risk of hypertension, especially in Caucasian population (Xie et al. 2017). On the other hand, in the study of Karbek et al. (2014) serum apelin levels were found significantly higher in patients with metabolic syndrome than age-matched control subjects and significantly associated with coronary atherosclerosis. The authors conclude that apelin can be used as a specific marker for insulin sensitivity in patients with metabolic syndrome. Studies in humans have shown an increase in plasma apelin levels in obesity, impaired glucose tolerance (IGT), and T2DM with a reduction in apelin levels accompanying weight loss following diet. These findings suggest the presence of resistance to apelin, in a similar fashion to insulin and leptin (Piya et al. 2013).

\section{Apelin and reproductive system}

The presence of the apelin system has been demonstrated in the human ovary. Expression of apelin and APNR in granulosa cells and oocytes are significantly increased with ovarian follicle size, decreased at the late luteal phase, and declined sharply during corpus luteum $(\mathrm{CL})$ regression. It is suggested that APLN and APLNR are involved in CL formation, function, and regression during the oestrous cycle. Meanwhile, progesterone has been demonstrated to stimulate the expression of APLNR in granulosa cells (Liu et al. 2020, Kurowska et al. 2018).

Reports regarding serum apelin levels in PCOS are controversial (Hasan et al. 2018, Liu et al. 2020, Nayaker et al. 2017). Apelin may contribute to the PCOS pathogenesis (Karimi et al. 2018). Apelin and APLNR participate in angiogenesis of the luteal phase, which is associated with follicular development. Luteal phase deficiency occurs due to insufficient progesterone produced by $\mathrm{CL}$. A dysfunction of CL results in infertility 
in PCOS. Hyperlipidaemia and IR result in CL dysfunction and a diminished ability of the CL to produce and secrete progesterone in women with PCOS. Because the expression of APLNR is stimulated by progesterone, a low level of progesterone might lead to a decrease in APLNR expression (Shirasuna et al. 2008). Recently, some studies have demonstrated that IGF-1 plays a significant role in the regulation and ovarian follicular maturation and steroidogenesis in patients with PCOS (Desai et al. 2015). Roche et al. (2016) in patients with PCOS observed that follicular fluid APLN concentration and granulosa cell APLN and APLNR mRNA expression was higher than that observed in control patients. In cultured human granulosa cells from non-obese patients without PCOS, insulin-like growth factor 1 (IGF1) increased APLNR expression, and recombinant human APLN (APLN-13 and APLN-17) increased both basal and IGF1-induced steroid secretion. IGF-1 promotes the production of oestrogen by stimulating APLN and APLNR. Patients with PCOS have been found to have low oestrogen which is a reason for the increase in APLN/APLNR in follicular fluid. IGF-1 action is modulated by IGFBP-1, which is synthesized in the liver. IGFBP-1 synthesis also occurs in ovarian granulosa cells and endometrium, and its synthesis is inhibited by insulin (Suikkari et al. 1988). The increased insulin levels reduce the synthesis of IGFBP-1, which in turn enhances the bioactivity of IGF-1. IGF-1 could stimulate oestrogen secretion both in granulosa and luteal cells, and IGF-2 stimulates the aromatization of androgens. Therefore, IGF-1 and APLN may exhibit a compensatory increase in PCOS (Hansen et al. 2014). Vascular endothelial growth factor (VEGF) is also critical for ovarian folliculogenesis and normal reproductive function. Serum VEGF concentrations in women with PCOS are significantly higher than those in women with healthy ovaries. VEGF could promote the accumulation of small follicles and increase cyst formation by binding to tyrosine kinase receptors. The APLN/APLNR system functions upstream of VEGF to promote ovarian angiogenesis and to change ovary morphology. However, it was found that hyperinsulinemia could stimulate APLN and VEGF expression: thus, insulin, apelin, and VEGF expression are increased in PCOS (Abd et al. 2005).

Choi and Altinkayia as well as others found that apelin levels were lower in PCOS than in healthy women with a positive correlation with BMI, insulin, HOMA-IR, triglycerides (TG), and free testosterone, speculating that apelin can be used as a marker for insulin sensitivity
(Hasan et al. 2018, Polak et al. 2017, Saleh et al. 2018). In the study of Choi et al. (2012), both PCOS groups (with/without hyperandrogenism) had significantly lower serum apelin levels compared with controls. Similarly, in the study of Altinkaya et al. (2014), serum apelin levels were lower in women with PCOS with BMI $\geq 25 \mathrm{~kg} / \mathrm{m}^{2}$ than in controls. Lower apelin levels were detected in normal weight women with PCOS as compared with BMI-matched controls. In this study, serum apelin levels were positively correlated with BMI, HOMA-IR, fasting glucose and insulin, TG, and free testosterone levels, but negatively correlated with HDL levels.

On the other side, some authors have found higher plasma apelin concentrations in patients with PCOS compared with healthy controls (Goren et al. 2012, Hasan et al. 2018, Polak et al. 2017, Liu et al. 2020). A study from Egypt demonstrated a significant increase in serum levels of apelin in non-obese and obese PCOS groups when compared to their controls. Within PCOS groups, there were significant positive correlations between apelin and both BMI as well as HOMA-IR (Abd-Elhafez et al. 2020). Either apelin levels in follicular fluid or its expression in granulosa cells were significantly higher in obese than in normal weight women and positively correlated with BMI. In another study, a positive correlation was found between follicle count and both apelin levels in follicular fluid and apelin expression in granulosa cells. Concerning APJ, the authors found the same significant results with a predominant expression of this receptor in the obese group and women with PCOS (Bongrani et al. 2019). On the other hand, APLN expression in follicular fluid was higher than its plasma levels in patients with PCOS, and the APLN concentrations in the follicular fluid were significantly higher in PCOS patients than it was in healthy controls. In addition, APLN and APLN expression in the granulosa cells of PCOS patients was significantly higher than in women without PCOS, and higher in obese PCOS than in non-obese subjects. The authors did not find any significant correlation between plasma apelin levels and biochemical or clinical data in PCOS women as well as control group, except for the negative correlation between apelin and SHBG levels in the control group (Goren et al. 2012).

Some authors reported an inverse association between apelin and glucose, insulin, and HOMA-IR values, supporting the role of apelin in the regulation of insulin sensitivity. Nutritional status seems to have different effects on apelin release in women with PCOS 
compared to those without PCOS. This may be caused by changes in leptin and resistin secretion and may enhance pituitary-ovarian axis disturbances. There were significantly lower plasma apelin-36 and -12 levels in obese PCOS patients than those in non-PCOS women. However, plasma apelin-36 and -12 levels were significantly higher in the normal weight PCOS subgroup as compared with the obese group (OlszaneckaGlinianowicz et al. 2013). The meta-analysis of 81 studies showed no significant changes in the circulating levels of apelin in non-obese PCOS relatives to non-obese healthy controls (Lin et al. 2021). Discrepant findings among the published studies may be attributed to differences in ethnicity, age, study design, genetic characteristics, and assessment methodology (Polak et al. 2017).

\section{Apelin and cardiovascular system in PCOS}

Being localized in human vascular and endocardial endothelial cells as well as smooth muscle cells of the vascular wall, apelin has been proposed to play a role in the CVS (Akinci et al. 2020). In patients with coronary heart disease apelin concentrations were reported to be lower than healthy controls, suggesting a potential role as an athero-protective factor. Overall, apelin has been described as a beneficial adipokine, and there has been speculation on whether it could be a promising therapeutic target in metabolic disorders and CVD (Aleksandrova et al. 2018). Subclinical atherosclerosis and chronic lowgrade vascular inflammation may occur more frequently among women without PCOS. However, it should be noted that the impaired endothelial function has also been observed independent of IR in women with PCOS. Therefore, the increased risk of CVD in women with PCOS might be partially attributed to hyperandrogenism (Kim et al. 2006, Kravariti et al 2005). Choi et al. (2012) investigated serum levels of CVD risk factors including apelin in non-obese women with PCOS. The study suggested that serum apelin levels were positively correlated with apolipoprotein A levels, which were regarded as an athero-protective marker and negatively with total testosterone independent of IR. Serum apelin levels were lower in women with PCOS compared with controls. Despite the presence of CVD risk factors there are currently no adequate prospective outcome data documenting the prevalence of $\mathrm{CV}$ events in PCOS. Several studies have established the functional and structural abnormalities of CVS in PCOS (Nayker et al. 2017).
Effect of pharmacologic treatment on serum apelin concentration in PCOS women

Sun et al. (2021) investigated the relevance of apelin and IR and the possible effect of the combined therapy of drospirenone-ethinylestradiol (DRSP-EE) combined with metformin in PCOS women. The PCOS group had higher apelin and fasting insulin levels, and HOMA-IR than the controls. After 3 months of combined treatment, the levels of apelin, testosterone, LH, LH/FSH, and fasting insulin, as well as HOMA-IR, decreased significantly. The levels of fasting glucose, cholesterol, TG, HDL, LDL, and FSH did not change significantly. Serum apelin concentration positively correlated with BMI, fasting insulin level, and HOMA-IR. BMI and HOMA-IR were independent factors related to the level of serum apelin. Less is known about the effect of metformin on serum apelin concentrations. Metformin is a biguanide used in the management of metabolic disorders and insulin resistance in PCOS. Although it may improve commonly associated metabolic and $\mathrm{CV}$ risk parameters and restore ovulation in women with PCOS, the effect on apelin levels is still controversial. Hasan and El Hameed (2018) found higher serum apelin levels in obese than lean PCOS women. Metformin treatment improved insulin sensitivity and decreased serum apelin, LH, and testosterone in both groups, although lean patients showed a better response to metformin treatment. These authors confirmed a significant positive correlation between serum apelin levels and BMI, serum insulin level, and HOMA-IR in all PCOS patients before and after metformin treatment. Probiotics are live microorganisms synergistic with the intestinal microbiota. There is evidence to verify the involvement of intestinal microbiota in IR, obesity, and low-grade inflammation, which are associated with PCOS. Therefore, it can be argued that symbiotic (probiotics and prebiotics) supplementation may potentially reduce the consequences of PCOS. In the randomized double-blind study of Karimi et al. (2018), authors examined the effect of synbiotics on serum apelin concentration and metabolic parameters in PCOS women. A 12 -week synbiotic supplementation had no significant beneficial effects on IR and hs-CRP in women with PCOS, whereas the serum levels of apelin 36 significantly decreased after treatment.

\section{Conclusion}

PCOS is the most common endocrine cause of 
menstrual irregularities, hirsutism, and acne, but its pathogenesis remains unclear. Insulin resistance and compensatory hyperinsulinemia play an important role in the pathogenesis of PCOS. Obesity is observed about $50-70 \%$ in patients with PCOS and mostly in the form of android obesity. Android type obesity and an increase in intraabdominal obesity were also shown in non-obese PCOS patients. Approximately $90 \%$ of obese women and $45 \%$ of lean women with PCOS have IR.

The apelinergic system was found in the hypothalamus, pituitary, ovaries, and testes, and has paracrine effects to control reproduction both in female and male regulation of their physiology. Apelin inhibits gonadotropin and PRL secretion in females and has a stimulatory effect on steroidogenesis and proliferation but an inhibitory action on cell apoptosis. There are limited data in the literature regarding the change in apelin levels and their relation to IR, obesity, and hormonal disturbances in PCOS. Study results regarding changes in apelin level or its relation to PCOS are inconsistent. Some studies reported lower levels of apelin in PCOS women, however, others reported higher levels. Choi et al. (2012) and Chang et al. (2011) reported a lower apelin level in normal-weight PCOS women than in the control group. These studies also suggest that serum apelin level is negatively correlated with total testosterone level and free androgen index (FAI) independently of IR. In contrast to these findings, other studies reported a higher level of apelin in PCOS than in controls. Similarly, Sun et al. (2015) and Abd-Elhafez et al. (2020) presented significantly higher serum apelin levels in PCOS women compared with controls. Moreover, apelin level was positively correlated with BMI, HOMA-IR, and fasting insulin level. Hasad and El Hameed (2018) showed significantly higher serum apelin levels in PCOS patients compared to lean ones. Higher levels of plasma apelin in PCOS might be related to androgenic obesity, increased WHR, increased adiposity, impairment in LH/FSH interaction, hypotalamohypophyseal axis effects and compensatory mechanisms due to the metabolic changes in PCOS. The implication of apelin in the pathogenesis of PCOS seems to be more complicated, involving disturbed synthesis of androgens. Larger studies may also aim to detect apelin levels in all phenotypes of PCOS considering the individual impacts of fat distribution, infertility, and hirsutism.

\section{Conflict of Interest}

There is no conflict of interest.

\section{Acknowledgements}

This work was supported by Ministry of Health 2019/32UPJŠ-4. Early diagnosis of cardiovascular disease using new hormonal biomarkers. Solution period: 2019-2022.

\section{References}

ABD-EL-AAL DE, MOHAMED SA, AMINE AF, MEKI A-R M A: Vascular endothelial growth factor and insulinlike growth actor-1 in polycystic ovary syndrome and their relation to ovarian blood flow. Eur J Obstet Gynecol Reprod Biol 118: 219-225, 2005. https://doi/10.1016/j.ejogrb.2004.07.024

ABD-ELHAFEZ ZZ, MOHAMED MA, ABD-ELMOUTTALEB AT, ABD-ELATIF DM: Evaluation of serum omentin-1 and apelin levels and their association with insulin resistance and obesity in Egyptian women with polycystic ovary syndrome. Int Res J Pharm11: 32-40, 2020. https://doi/10.7897/2230-8407.110216

AKINCI B, CELTIK A, TUNALI S, GENC S, YUKSEL F, SECIL M, OZCAN MA, BAYRAKTAR F: Circulating apelin levels are associated with cardiometabolic risk factors in women with previous gestational diabetes. Arch Gynecol Obstet 289: 787-793, 2014. https://doi/10.1007/s00404-013-3070-y

ALEKSANDROVA K, MOZAFFARIAN D, PISCHON T: Addressing the perfect storm: biomarkers in obesity and

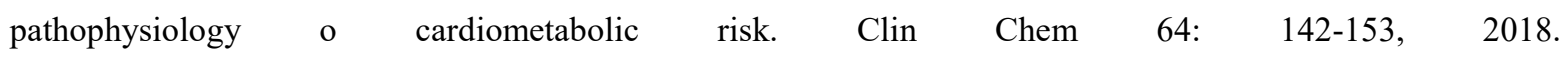
https://dx.doi.org/10.1373/clinchem.2017.275172

ALTINKAYA SO, NERGIZ S, KUCUK M, YUKSEL H: Apelin levels in relation with hormonal and metabolic profile in patients with polycystic ovary syndrome. Eur J Obstet Gynecol Reprod Biol 176: 168-172, 2014. https://dx.doi.org/10.1016/j.ejogrb.2014.02.022

BONGRANI A, MELLOUK N, RAME CH, CORNUAU M, GUÉRIF F, FROMENT P, DUPONT J: Ovarian expression of adipokines in polycystic ovary syndrome: a role for chemerin, omentin, and apelin in follicular growth arrest and ovulatory dysfunction? Int J Mol Sci 20: 1-22, 2019. https://doi/10.3390/ijms.20153778 
CEKMEZ F, CEKMEZ Y, PIRGON O, CANPOLAT FE, AYDINOZ S, IPCIOGLU OM, KARADEMIR F: Evaluation of new adipocytokines and insulin resistance in adolescents with polycystic ovary syndrome. Eur Cytokine Netw 22: 32-37, 2011. https://doi/10.1684/ecn.2011.00.279

CHANG CY, TSAI YC, LEE CH, CHAN TF, WANG SH, SU JH: Lower serum apelin levels in women with polycystic ovary syndrome. Fertil Steril 95: 2520-2523, 2011. https://doi/10.1016/fertnstert.2011.04.044

CHOI YS, YANG HI, CHO SH, JUNG JA, JEON YE, KIM HY, SEO SK, LEE BS: Serum asymmetric dimethylarginine, apelin, and tumor necrosis factor- $\alpha$ levels in non-obese women with polycystic ovary syndrome. Steroids 77: 1352-1358, 2012. https://dx.doi.org/10.1016/j.steroids.2012.08.005

DESAI A, PATEL SS: Increased insulin-like growth factor-1 in relation to cardiovascular function in polycystic ovary syndrome: friend or foe? Gynecol Endocrinol 31: 801-807, 2015. https://doi/10.3109/09513590.2015.1075497

GOREN K, SAGSOZ N, NOYAN V, YUCEL A, CAGLAYAN O, BOSTANCI MS: Plasma apelin levels in patients with polycystic ovary syndrome. J Turkish-German Gynecol Assoc 13: 27-31, 2012. https://doi/10.5152/jtgga.2011.74

HANSEN KA: Polycystic ovary syndrome. S D Med 67: 443, 2014.

HASAN MM, ABD EL HAMMED AA: Serum adipokine (apelin) in lean and obese polycystic ovary syndrome patients before and after metformin treatment. Middle East Fertility Society J 23: 315-318, 2018. https://doi.org/10.1016/j.mefs.2018.04.003

KARBEK B, BOZKURT NC, TOPALOGLU O, ASLAN MS, GUNGUNES A, CAKAL E, DELIBASI T: Relationship of vaspin and apelin levels with insulin resistance and atherosclerosis in metabolic syndrome. Minerva Endocrinol 39: 99-105, 2014.

KARIMI E, MOINI A, YASERI M, SHIRZAD N, SEPIDARKISH M, HOSSEIN-BOROUJERDI M, HOSSEINZADEH-ATTAR MJ: Effects of synbiotic Supplementation on metabolic parameters and apelin in women with polycystic ovary syndrome: a randomized double-blind placebo-controlled trial. Brit J Nutrition 119: 398-406, 2018. https://doi/10.1017/S0007114517003920

KIM JA, MONTAGNANI M, KOH KK, QUON MJ: Reciprocal relationships between insulin resistance and endothelial dysfunction: molecular and pathophysiological mechanisms. Circulation 113: 1888-1904, 2006. https://doi/10.1161/CIRCULATIONAHA.105.563213

KRAVARITI M, NAKA KK, KALANTARIDOU SN, KAZAKOS N, KATSOURAS CS, MAKRIGIANNAKIS A, PARASKEVAIDIS EA, CHROUSOS GP, TSATSOULIS A, MICHALIS LK: Predictors of endothelial dysfunction in young women with polycystic ovary syndrome. J Clin Endocrinol Metab 90: 5088-5095, 2005. https://doi/10.1210/jc.2005-0151

KUROWSKA P, BARBE A, ROZYCKA M, CHMIELINSKA J, DUPONT J, RAK A: Apelin in reproductive physiology and pathology of different species: a critical review. Int J Endocrinol 9170480, 2018. https://dx.doi.org/10.1155/2018.9170480

LIN K, SUN X, WANG X, WANG H, CHEN X: Circulating adipokine levels in nonobese women with polycystic ovary syndrome and in nonobese control women: a systematic review and meta-analysis. Front Endocrinol 11: article 537809, 2021. https://doi/10.3389/fendo.2020.537809

LIU Q, JIANG J, SHI Y, MO Z, LI M: Apelin/Apelin receptor: A new therapeutic target in Polycystic Ovary Syndrome. Life Sciences 260: 118310, 2020. https://doi.org/10.1016/j.1fs.2020.118310

Nayaker BS, Thomas S, Ramachandran S, Loganathan S, Sundari M, Mala K. Polycystic ovarian syndrome-associated cardiovascular complications: An overview of the association between the biochemical markers and potential strategies for their prevention and elimination. Epub 2017 Jul 8. PMID: 28711514.

NAYAKER BS, THOMAS S, RAMACHANDRAN S, LOGANATHAN S, SUNDARI M, MALA K: Polycystic ovarian syndrome-associated cardiovascular complications: An overview of the association between the biochemical markers and potential strategies for their prevention and elimination. Diabetes Metab Syndr 11 Suppl 2: S841-S851, 2017. https://doi/10.1016/j.dsx.2017.07.004

OLSZANECKA-GLINIANOWICZ M, MADEJ P, NYLEC M, OWCZAREK A, SZANECKI W, SKALBA P, CHUDEK J: Circulating apelin level in relation to nutritional status in polycystic ovary syndrome and its association with metabolic and hormonal disturbances. Clin Endocrinol 79: 238-242, 2013. https://doi/10.1111/cen.12120 
PIYA MK, MCTERNAN PG, KUMAR S: Adipokine inflammation and insulin resistance: the role of glucose, lipids, and endotoxin. Journal of Endocrinology 216: 1-15, 2013. https://doi/10.1530/JOE-12-0498

POLAK K, CZYZYK A, SIMONCINI T, MECZEKALSKI B: New markers of insulin resistance in polycystic ovary syndrome. J Endocrinol Invest 40: 1-8, 2017. https://doi/10.1007/s40618-016-0523-8

ROCHE J, RAMÉ CH, REVERCHON M, MELLOUK N, CORNUAU M, GUERIF F, FROMENT P, DUPONT J: Apelin (APLN) and Apelin Receptor (APLNR) in human ovary: expression, signalling, and regulation of steroidogenesis in primary human luteinized granulosa cells. Biol Reprod 95: 104, 2016. https://doi/10.1080/1095/biolreprod.116.141754

SALEH IBRAHIM MK, IBRAHIM MOSTAFA M, MOHAMAD ABDELLA R, ALI MARZOUK S, EL-ATTAR S: Relation of serum apelin levels to ultrasound in images and Doppler index in diagnosed Polycystic ovary syndrome in overweight and obese women. Middle East Fertility Society J 23: 377-383, 2018. https://doi.org/10.1016/j.mefs.2018.05.007

SHIRASUNA K, SHIMIZU T, SAYAMA K,ASAHI T, SASAKI M, BERISHA B, SCHAMS D, MIYAMOTO A: Expression and localization of apelin and its receptor APJ in the bovine corpus luteum during the estrous cycle and prostaglandin F2 alpha-induced luteolysis. Reproduction 135: 519-525, 2008. https://doi/10.1530/REP-07$\underline{0409}$

SUIKKARI AM, KOIVISTO VA, RUTANEN EM, YKI-JÄRVINEN H, KARONEN SL, SEPPÄLÄ M: Insulin regulates the serum levels of low molecular weight insulin-like growth factor-binding protein. J Clin Endocrinol Metab 66: 266-272, 1988. https://doi/10.1210/jcem-66-2-266

SUN X, WU X, ZHOU Y, YU XINYAN, ZHANG W: Evaluation of apelin and insulin resistance in patients with PCOS and therapeutic effect of drospirenone-ethinylestradiol plus Metformin. Med Sci Monit 21: 2547-2552, 2015. https://doi/10.12659/MSM.894926

XIE H, LUO G, ZHENG Y, HU D, PENG F, XIE L: Lowered circulating apelin is significantly associated with an increased risk for hypertension: a meta-analysis. Clin Exp Hypertens 39: 435-401, 2017. https://doi/10.1080/10641963.2016.1267199 